(C) 1983. The Genetical Society of Great Britain

\title{
JOINT ANALYSIS OF TRIPLE TEST CROSSES OF A SINGLE POPULATION WITH ASSOCIATED AND DISPERSED INBRED TESTERS
}

\author{
H. S. POONI AND J. L. JINKS \\ Department of Genetics, University of Birmingham, Birmingham B15 2TT, UK
}

Received 12.i.83

\begin{abstract}
SUMMARY
If two pairs of pure breeding lines and their respective $F_{1}$ 's are used as two sets of testers in triple test cross investigations of the same population, the data can be subjected to a combined orthogonal analysis of variance. Where one pair of pure breeding testers is relatively dispersed (usually the parents whose cross produced the population) and the other pair is relatively associated (usually extreme selections from the population), the orthogonal comparisons yield estimates of the additive genetic, dominance and epistatic components of variation and information about the genetical constitution of the testers.

The theoretical expectations for the six principal orthogonal comparisons of the combined analysis are presented for the simple case which assumes no non-allelic interaction and a linkage equilibrium, and for more complex situations where one or both of these assumptions is relaxed. The combined analysis of two triple test crosses on $60 \mathrm{~F}_{\infty}$ families derived from the cross of V2 and V12 of Nicotiana rustica, using V2, V12 and their $F_{1}$ as one set of testers and two extreme $F_{\infty}$ families, D10 and D17 and their $F_{1}$ as the other set, confirm earlier analyses of this cross. The estimates of the genetical components are intermediate in value between those of the separate, conventional triple test cross analyses but the standard errors of the dominance components are lower. In addition the combined analyses show that there have been major changes in the distribution of dominant alleles between the two sets of inbred testers as well as changes in the phases of alleles in the testers at loci that contribute to the epistatic variation. These changes are in the direction expected from the origins of the testers and their estimated coefficients of association/dispersion.
\end{abstract}

\section{INTRODUCTION}

In a previous paper (Pooni and Jinks, 1983) the consequences of using associated as opposed to dispersed inbred testers $\left(\mathrm{L}_{1}\right.$ and $\left.\mathrm{L}_{2}\right)$ in a triple test cross were examined theoretically and experimentally in material from the cross of varieties 2 and 12 of Nicotiana rustica. While only one set of inbred testers and their $F_{1}$ is required for a triple test cross, in this paper we shall examine the additional information that can be obtained when both relatively dispersed and associated pairs of inbred testers and their $F_{1}$ 's are used simultaneously to investigate the same population.

\section{THEORY}

With associated (a) and dispersed (d) pairs of inbred testers ( $\mathrm{L}_{1}$ and $L_{2}$ ) and their respective $F_{1}$ 's $\left(L_{3}\right.$ or $L_{3}$ and $R L_{3}$ if reciprocals are used) six (or eight with reciprocals) orthogonal comparisons can be made among the $n$ groups of six (or eight) families produced by crossing each plant or family 
of the $n$ under test with each tester. For the $i$ th group these comparisons are:

Without reciprocal $F_{1}$ testers

\begin{tabular}{ccccccc} 
Comparison & \multicolumn{3}{c}{ Dispersed testers } & \multicolumn{3}{c}{ Associated testers } \\
& $\mathrm{d}_{1 \mathrm{~L}}$ & $\mathrm{~d} \overline{\mathrm{L}}_{2 \mathrm{i}}$ & $\mathrm{d} \overline{\mathrm{L}}_{3 \mathrm{i}}$ & $\mathrm{a} \overline{\mathrm{L}}_{1 \mathrm{i}}$ & $\mathrm{a} \overline{\mathrm{L}}_{2 \mathrm{i}}$ & $\mathrm{a} \overline{\mathrm{L}}_{3 \mathrm{i}}$ \\
1 & +1 & +1 & +1 & +1 & +1 & +1 \\
2 & +1 & +1 & +1 & -1 & -1 & -1 \\
3 & +1 & -1 & & +1 & -1 & \\
4 & +1 & -1 & & -1 & +1 & \\
5 & +1 & +1 & -2 & +1 & +1 & -2 \\
6 & +1 & +1 & -2 & -1 & -1 & +2
\end{tabular}

With reciprocal $F_{1}$ testers

$\begin{array}{ccccccccc} & \mathrm{d}_{\mathrm{L}}^{1 \mathrm{i}} & \mathrm{d} \overline{\mathrm{L}}_{2 \mathrm{i}} & \mathrm{d} \overline{\mathrm{L}}_{3 \mathrm{i}} & \mathrm{d} R \overline{\mathrm{L}}_{3 \mathrm{i}} & \mathrm{a} \overline{\mathrm{L}}_{1 \mathrm{i}} & \mathrm{a} \overline{\mathrm{L}}_{2 \mathrm{i}} & \mathrm{a} \overline{\mathrm{L}}_{3 \mathrm{i}} & \mathrm{a} R \overline{\mathrm{L}}_{3 \mathrm{i}} \\ 1 & +1 & +1 & +1 & +1 & +1 & +1 & +1 & +1 \\ 2 & +1 & +1 & +1 & +1 & -1 & -1 & -1 & -1 \\ 3 & +1 & -1 & & & +1 & -1 & & \\ 4 & +1 & -1 & & & -1 & +1 & & \\ 5 & +1 & +1 & -1 & -1 & +1 & +1 & -1 & -1 \\ 6 & +1 & +1 & -1 & -1 & -1 & -1 & +1 & +1 \\ 7 & & & +1 & -1 & & & +1 & -1 \\ 8 & & & +1 & -1 & & & -1 & +1\end{array}$

Leaving aside comparisons 7 and 8 which detect differences between reciprocal $F_{1}$ testers ascribable to the usual causes of differences between reciprocal crosses (Mather and Jinks, 1971), comparisons 1, 3 and 5 are the standard ones for the additive genetic, dominance and epistatic components of variation, respectively, summed over the two triple test crosses. The remaining three comparisons, 2, 4 and 6 are the standard ones for the same three genetical components differenced over the two triple test crosses.

The expectations for comparisons 1 to 6 have been obtained for a single pair of loci at which the alleles are associated (AABB and aabb) and dispersed (AAbb and $\mathrm{a} a \mathrm{BB}$ ) in the two sets of inbred testers respectively. The populations under test are the $F_{2}$ and $F_{\infty}$ generations of a cross between two pure breeding lines in which the alleles are dispersed. Four levels of complexity of the genetical variation are considered:

1. No epistasis and linkage equilibrium.

2. No epistasis and linkage disequilibrium.

3. Epistasis and linkage equilibrium.

4. Epistasis and linkage disequilibrium.

As in the previous paper (Pooni and Jinks, 1983) all the expectations have been adjusted to make them directly comparable. The expectations for comparisons 1,3 and 5 are given in table 1 and those for comparisons 2,4 and 6 in table 2 , for the case where reciprocal $\mathrm{F}_{1}$ testers $\left(\mathrm{L}_{3}\right.$ and $\left.R \mathrm{~L}_{3}\right)$ are available.

Irrespective of whether we assume the simplicity of model 1 or allow for the greater complexities of models 2, 3 and 4, all of the additive genetic contribution to the variance goes into comparison 1 and none into comparison 2. In the presence of epistasis (models 3 and 4), however, the 
TRIPLE TEST CROSSES

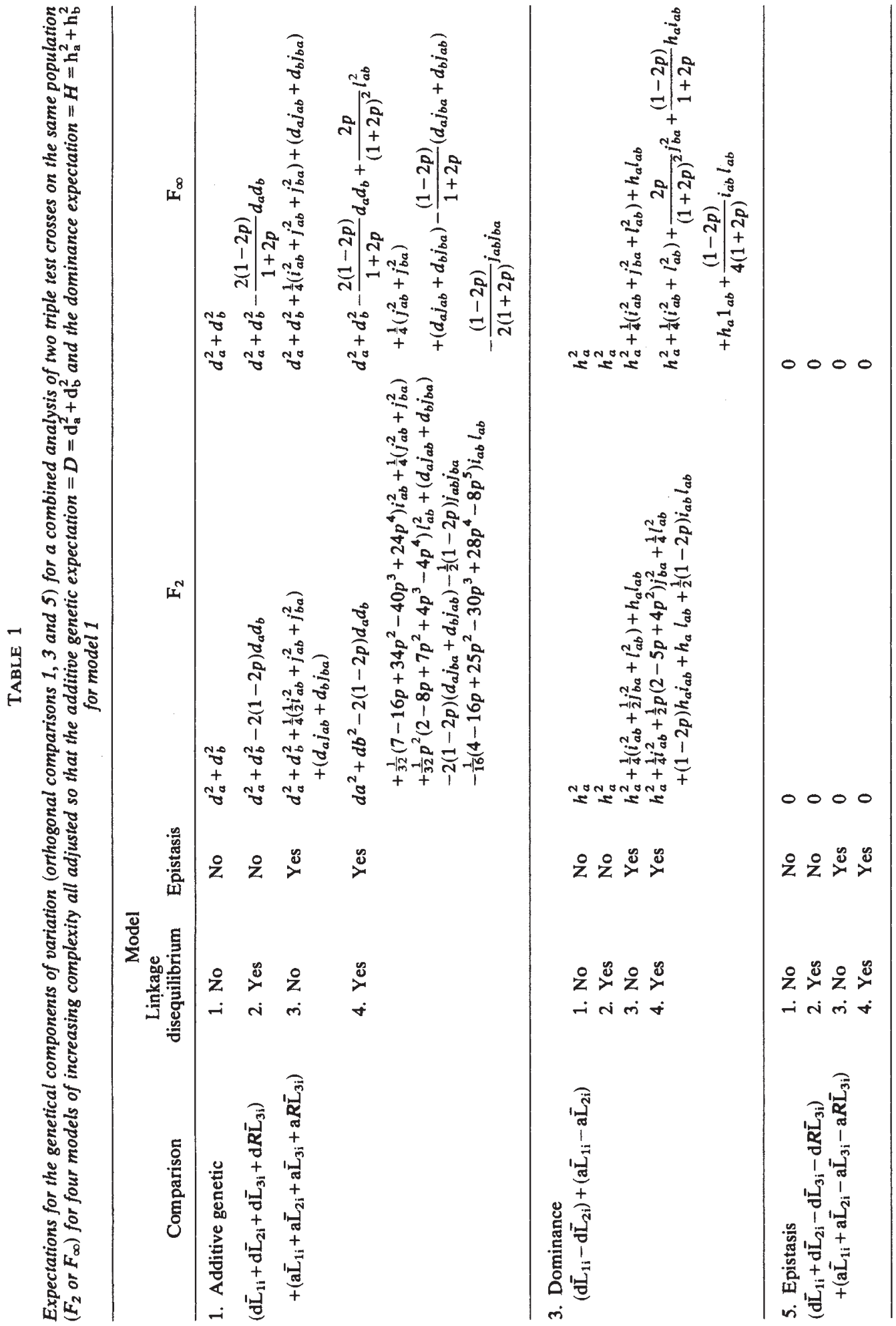




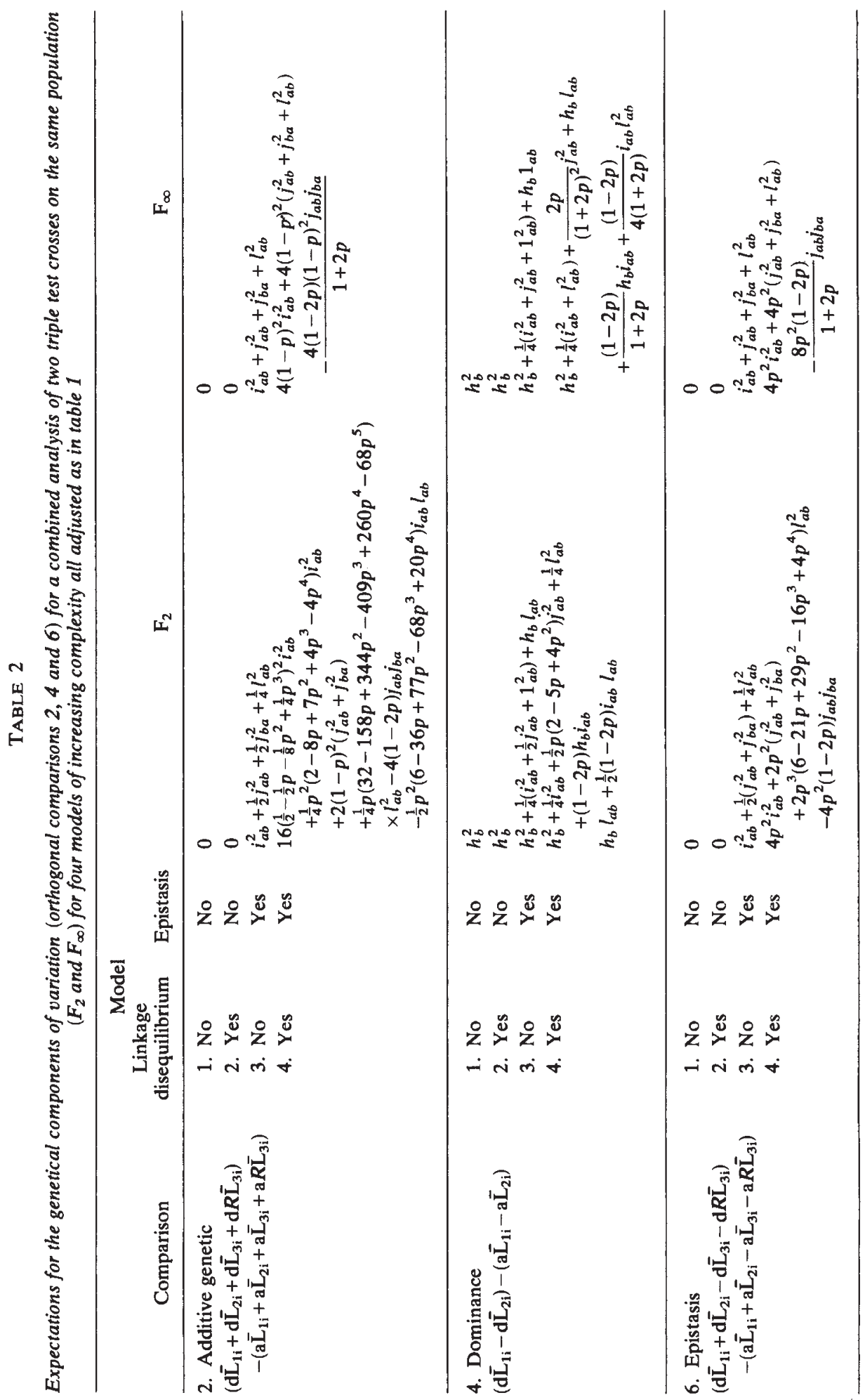


epistatic bias on the additive genetic component appears partly in comparison 1 and partly in comparison 2 . As a consequence comparison 2 for models 3 and 4 measures purely epistatic variation and, therefore, provides an additional test for its presence.

In contrast, the dominance contribution to the variance is split between comparisons 3 and 4 for all four models. The dominance contribution of loci whose alleles are distributed in the same way between the $L_{1}$ and $L_{2}$ testers in both the associated and dispersed sets, that is, $L_{1}$ is $A A$ and $L_{2}$ is aa for both sets of testers, appears in comparison 3. The contribution of loci whose alleles are distributed in the opposite way between the $L_{1}$ and $L_{2}$ testers in the associated and dispersed sets, that is $L_{1}$ is $B B$ and $L_{2}$ is bb in one set and $L_{1}$ is $b b$ and $L_{2}$ is $B B$ in the other set, appears in comparison 4 . The epistatic bias on the dominance component in models 3 and 4 is also split between comparisons 3 and 4 on the same basis.

The epistatic comparisons, 5 and 6 , differ yet again in that comparison 5 has an expectation of zero for all four models; for models 1 and 2 because there is no epistasis and for models 3 and 4 because the epistatic contributions of the associated and dispersed sets of testers cancel out. For comparison 6 there is an epistatic contribution for models 3 and 4 . It should be noted that comparisons 2 and 6 for model 3 have exactly the same expectation (table 2).

Extension of these expectations to many pairs of loci introduces no new principles but modifies points of detail. In the straightforward case where there is complete dispersion in the dispersed testers $\left(r_{\mathrm{d}}=0\right)$ and complete association in the associated testers $\left(r_{\mathrm{d}}=1\right)$, the increasing allele at loci responsible for half of the additive genetic effects in the $\mathrm{L}_{1}$ tester of the associated pair will have been substituted for the decreasing allele in the $\mathrm{L}_{1}$ tester of the dispersed pair. Hence, half the alleles in the $\mathrm{L}_{1}$ tester of the associated and dispersed pairs will be the same and half different. The dominance contribution would, therefore, be expected to be divided approximately equally between comparisons 3 and 4 . We should note, however, that differences in the distribution of alleles between the $\mathrm{L}_{1}$ and $\mathrm{L}_{2}$ testers of the associated and dispersed pairs do not necessarily contribute to differences in the degree of association/dispersion $\left(r_{\mathrm{d}}\right)$. For example, if at the $A$, a and $B, b$ loci $L_{1}$ is $A A b b$ and $L_{2}$ aaBB in the dispersed pair and $L_{1}$ is aaBB and $L_{2} A A b b$ in the associated pair these loci will not contribute to any change in $r_{d}$ but their dominance contributions will be made exclusively to comparison 4 . There is, therefore, no direct relationship between the relative values of $r_{d}$ in the associated and dispersed pairs of testers and the relative contributions of the dominance component to comparisons 3 and 4.

For many pairs of interacting gene loci comparison 5 for models 3 and 4 is no longer necessarily zero. Thus it will be zero only if every pair of interacting genes which is dispersed in one pair of $L_{1}$ and $L_{2}$ testers is associated in the other pair and vice versa. Any pair of genes which is in the same phase, associated or dispersed, in both pairs of testers will contribute to comparison 5 but not to comparison 6 , while any pair of genes, which is in the opposite phase will contribute to comparison 6 but not to comparison 5 .

In general the dispersed set of testers will be the parents and $F_{1}$ of the cross from which the $F_{2}$ and $F_{\infty}$ populations under test will have been 
derived while the associated set will be the extreme phenotypes of the $F_{\infty}$ and their $F_{1}$ cross. This guarantees that the dispersed testers will differ for the same alleles at the same loci as are segregating in the population under test which is a requirement for the standard analysis and interpretation of triple test crosses. While the choice of extreme phenotypes from the $F_{\infty}$ maximises the probability of this requirement being met in the associated testers, it does not guarantee it and by chance the $L_{1}$ and $L_{2}$ testers may have fixed the same allele at some of the loci with consequences that are well known (Virk and Jinks, 1977; Jinks and Virk, 1977). The effect of the resulting inadequacy of the testers on the various comparisons is, however, relatively small because the proportion of fixed loci is itself usually small and while they are fixed in the testers they are still segregating in the population under test.

\section{Material}

The material is identical to that described by Pooni and Jinks (1983) except that six characters viz, three heights taken 2, 4 and 6 weeks after transplanting into the field $\left(\mathrm{H}_{1}, \mathrm{H}_{2}\right.$ and $\left.\mathrm{H}_{3}\right)$, height at flowering time (HFT) and length and width of the largest leaf (LL and LW), in addition to final height (FH) and flowering time (FT), will be analysed to increase the range of combinations of $r_{\mathrm{d}}$ values in the dispersed and associated sets of testers. The $r_{\mathrm{d}}$ values of the V2 and V12 and D10 and D17 testers used in the present experiment have been given by Pooni and Jinks (1981) and are repeated below for convenience.

$\begin{array}{ccccccccc}\text { Testers } & \mathrm{H}_{1} & \mathrm{H}_{2} & \mathrm{H}_{3} & \text { FT } & \text { HFT } & \text { LL } & \text { LW } & \text { FH } \\ \text { V2 and V12 } & 0.45 & 0.82 & 0.95 & 0.59 & 0.21 & 0.13 & 0.25 & 0.35 \\ \text { D10 and D17 } & 0.82 & 0.74 & 0.35 & 0.85 & 0.82 & 0.84 & 0.96 & 0.94\end{array}$

The proportions of decreasing and increasing alleles in the higher scoring $\left(\mathrm{L}_{1}\right)$ tester and therefore of decreasing and increasing alleles in the lower scoring $\left(\mathrm{L}_{2}\right)$ tester for any character can be obtained from these $r_{\mathrm{d}}$ values as $\left(1-r_{\mathrm{d}}\right) / 2$ and $\left(1+r_{\mathrm{d}}\right) / 2$ respectively. For instance for final height these proportions are 0.03 and 0.97 for D17 and 0.97 and 0.03 for D10.

\section{RESUlts}

The mean squares corresponding with the six orthogonal comparisons and their respective errors for each of the eight characters are given in table 3. With one exception, that is, comparison 6 for $\mathrm{LL}$, all mean squares are significant $(P<0 \cdot 05)$. Furthermore, with the exception of comparison 2 , the difference comparisons (4 and 6 ) are the same order of magnitude as the corresponding sum comparisons ( 3 and 5 , respectively).

As expected from previous analyses of derivatives of the cross of varieties 2 and 12 (Pooni and Jinks, 1981; Pooni, Jinks and Jayasekara, 1978) there is significant epistasis for all characters (comparisons 5 and 6 , table 3). In general, comparison 5, which is the contribution of interactions at loci whose alleles have retained the same phase (association or dispersion) in the two sets of testers, is of the same order of magnitude as comparison 6 , which is the contribution of interactions at loci whose alleles have reversed their phase between the two sets of testers. It is noteworthy that 
TRIPLE TEST CROSSES

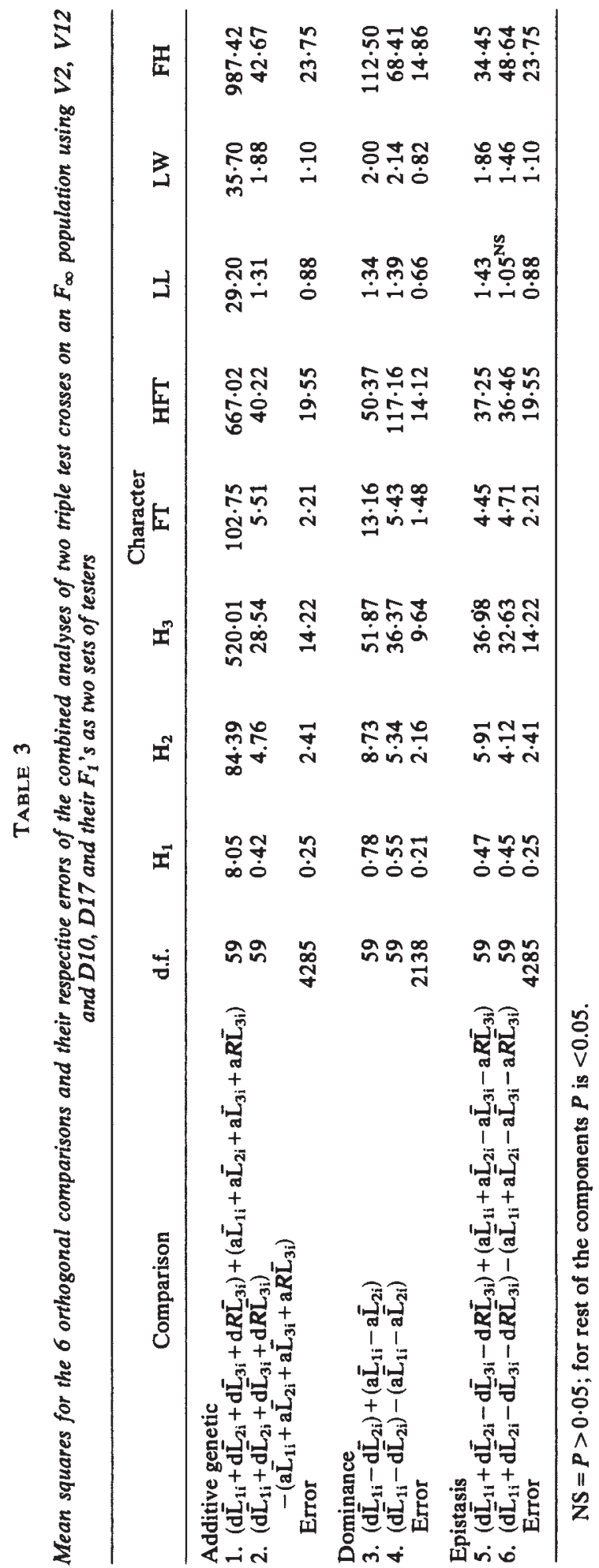


FT and FH, the two characters of primary concern when the testers D10 and D17 were chosen as extremes, show the highest proportion of contributions from loci where there has been a change of phase (comparison $6>$ comparison 5 , table 3 ). A further point of interest is the clear confirmation of the theoretical expectation (tables 1 and 2) that in the presence of epistasis comparisons 2 and 6 would be independent estimates of the same epistatic components of variation.

Comparisons 3 and 4 (table 3 ) confirm the presence of dominance for all characters. With the exception of HFT, comparison 3, which is the dominance contribution of loci where the same allele is present in the $\mathrm{L}_{1}$ tester of both sets, is greater than comparison 4 , which is the dominance contribution of loci where the alternative alleles are present in the $L_{1}$ testers of the two sets. The total dominance contribution of all loci segregating in the population is obtained from the sum of the mean squares from comparisons 3 and 4 . Comparison 1 provides the corresponding additive genetic contribution.

The estimates of the additive genetic (D) and dominance $(\mathrm{H})$ components of variation are present in table 4 along with those from the two independent conventional triple test cross analyses. For every character the combined estimate falls between the two independent estimates in magnitude and the combined estimates of $\mathrm{H}$ have smaller standard errors.

\section{Conclusions}

The theory presented in section 2 and the results of its application to an $\mathrm{F}_{\infty}$ population in section 4 shows that a combined orthogonal analysis of two triple test crosses can be used to detect and estimate the additive genetic, dominance and epistatic components of variation. The combined analysis is applicable only where each individual or family in the sample drawn from the population is crossed to all six (or eight) testers. It cannot, therefore, be used to compare two different populations by crossing them to the same or different sets of testers. The estimates of the genetical components from the combined analysis are intermediate in value between those from the separate triple test cross analyses (table 4 ) but the estimates of the dominance components have smaller standard errors.

The additional information the combined analysis provides over and above either of the single triple test crosses relates almost entirely to the properties of the testers. Thus comparisons 3 and 4 tell us about the relative distributions of alleles in the two sets of testers at loci that contribute to the dominance components while comparisons 5 and 6 tell us about the changes in phase of alleles in the testers at the pairs of loci that contribute to the epistatic components. Comparison 2 provides an independent estimate of the epistatic component of comparison 6 . The new information that emerges from the analysis of the $F_{\infty}$ population confirms the major redistribution of alleles between the V2 and V12, and D10 and D17 pairs of inbred testers that was expected from their different origins and $r_{\mathrm{d}}$ values (section 3).

From the practical point of view the most encouraging result is the robustness of the estimates of D (table 4 ) which do not differ significantly over the three sources of estimates for any character. This is no more than was expected for FH and FT since D10 and D17 were chosen to be extreme 


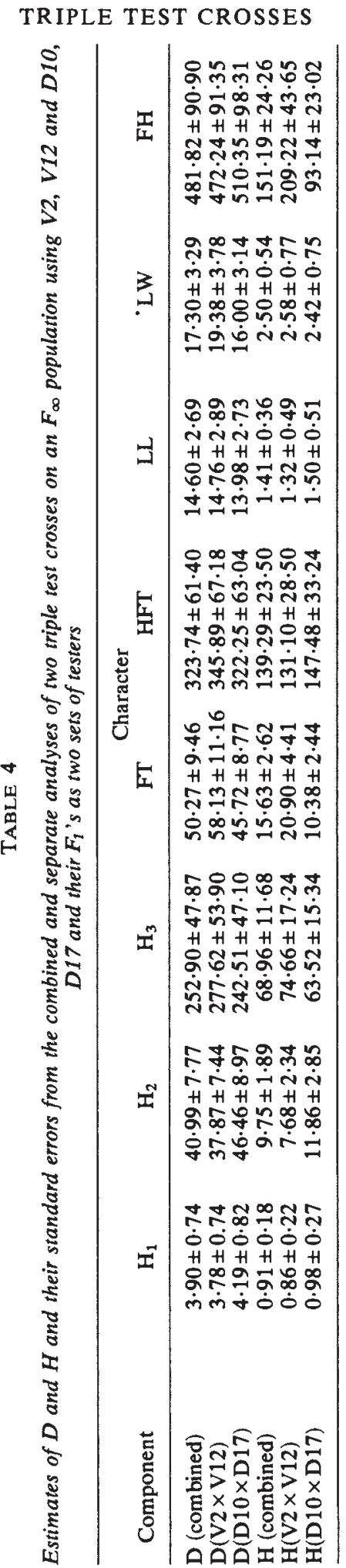


combinations for these characters but it is equally true for characters such as $\mathrm{H}_{2}$ and $\mathrm{H}_{3}$ for which D10 and D17 are less extreme than V2 and V12.

\section{REFERENCES}

JINKS, J. L. AND VIRK, D. S. 1977. A modified triple test cross analysis to list and allow for inadequate testers. Heredity, 39, 165-170.

MATHER, K. AND JINKS, J. L. 1971. Biometrical Genetics, Second edition. Chapman and Hall, London.

POONI, H. S. AND JINKS, J. L. 1981. The true nature of the non-allelic interactions in Nicotiana rustica revealed by association crosses. Heredity, 47, 253-258.

POONI, H. S. AND JINKS, J. L. 1983. Comparison of associated and dispersed testers in the triple test cross. Heredity, 50, 73-83.

POONI, H. S., JINKS, J. L. AND JAYASEKARA, N. E. M. 1978. An investigation of gene action and genotype $x$ environment interaction in two crosses of Nicotiana rustica by triple test cross and inbred line analysis. Heredity, 41, 83-92.

VIRK, D. S. AND JINKS, J. L. 1977. The consequences of using inadequate testers in the simplified triple test cross. Heredity, 38, 237-251. 